\title{
Thoracic radiation therapy: dosage and techniques
}

\author{
Andrew T. Turrisi, III \\ Department of Radiation Oncology, The University of Michigan Medical School, Ann Arbor, MI 48109-0010 (U.S.A.)
}

(Accepted 18 June 1989)

Key words: Small cell lung cancer, Radiotherapy to chest; Radiotherapy variably; Local control; Local failure

Combination chemotherapy (CC) deserves credit for the increase in survival for patients with limited small cell lung cancer (SCLC). However, the best CC is not clearly established. The definition of limited disease and the work-up to define limited disease has evolved over the last 20 years. Imaging studies are now more sophisticated (MRI and CT scan) and used more frequently. Although there is general agreement on what constitutes limited disease and what is not, the extent of the staging work-up, the issue of ipsilateral pleural effusion and supraclavicular disease continue to stimulate lively discussion.

The last decade's studies usually employed cyclophosphamide-based CC. Presently, platinum-etoposide (PE) CC is assuming increased importance at least in North America. However, in limited disease, no data compare the regimens directly.

Integration of CC with thoracic radiotherapy (TRT) has warranted much attention. These issues have been addressed in 3 recent reviews [1-3]. Three randomized North American studies, the SEG [4], the NCI-USA [5], and the CALGB [6], all support a survival benefit for the group receiving TRT. However, the British Medical Research Council (MRC) [7], the Finsen Institute [8] and the later Southeastern Cancer Study Group (SEG) trial did not show improvement for the TRT group. For all of these trials, the CC was cyclophosphamide-based, but the radiotherapy varied in many ways.

TRT has been known to produce dramatic regression of primary tumors in SCLC. The failure of CC to durably control the bulk primary location and TRT's local efficacy both suggested their combination. Comis reported local failure in $50-100 \%$ of cases [9].

The method of CC-TRT is another factor that influences toxicity for sure and may also be important in efficacy and survival, but the available information prevents reaching conclusions.

There are major problems that interfere with retrospective literature analysis of this issue. The term 'local control' is quite imprecise. Assessment of local control in most series has been based on clinicians' assessment of chest X-rays. More recently bronchoscopy and CT-scanning

Correspondence: A.T. Turrisi III, Department of Radiation Oncology, University of Michigan, Medical School, 1500 East Medical Center Drive, Ann Arbor, MI 48109-0010, U.S.A.

0169-5002 / 89/\$03.50 @ 1989 Elsevier Science Publishers B.V. (Biomedical Division) 
have also been included. However, selected cases have been subjected to these expensive and invasive tests. Moreover, definition of 'chest failure' commonly includes distant chest sites contralateral lung, ipsilateral, parenchymal, and pleural-based densities or effusions. Furthermore, in patients failing at distant sites, attention to local control or failure was likely to be less carefully detailed. Separating the evaluation of failure pattern into 'lung only' and 'local plus distant', if accurate, focuses on whether better local treatment or better systemic treatment is more important. Those with systemic failure have no prospect for improvement with local modalities.

Local failure data commonly report failure from response. It is not clear how patients with less-than complete response are handled. When treatment regimens have varied response rates, the proportion failing initial treatment and the proportion that fail after initial response are quite different. The term 'local control' is applied to all patients, but 'local failure' is commonly applied only after failure from response, but these terms are not used uniformly. Furthermore, studies with short median survival or few 2-year survivors may over-estimate local control. Determination of local status can be quite subjective in patients receiving TRT. Thus, future trials need to study the endpoint of local control recognizing the above pitfalls and then design studies that can clarify these issues.

If the issue of excessive local failure is important, optimizing TRT may yield results. Defining (1) volume, (2) course, (3) dose, (4) duration of therapy, as well as integration with systemic therapy are all issues that have received surprisingly little attention. These variables certainly may influence local control and possibly survival. Table 1 presents the local control reported from 10 recent studies.

These studies were selected because they reported local failure or control. In the 4 controlled studies, TRT reduced local failure on each study. With CC alone local failure ranged between $40 \%$ and $82 \%$; with the addition of TRT it ranged between $18 \%$ and $69 \%$.

\section{Volume, course, dose}

Pre-chemotherapy vs post-chemotherapy TRT volume is a topic of discussion in other diseases. For concurrent methods, the initial volume is the pre-chemotherapy volume. With the alternating strategy, the subsequent courses can often have reduced volumes, but no study reports the influence of either maintaining a large volume or reducing it after response. The sequential method is the setting to evaluate this. Using larger volumes versus smaller or tighter volumes is another issue. Understanding the physics of field borders and the lymphatic spread of lung tumors has caused TRT field sizes to increase. Radiating larger field sizes necessarily increases dose to normal tissues, which potentially increases toxicity, particularly in conjunction with CC. Table 2 demonstrates the influence of larger versus smaller volumes as described in these studies. Custom shields or blocks and techniques to maximize tumor dose and minimize nomal tissue dose warrant attention. Posterior spinal shields inadvertently protect the mediastinum, and this may cause local failure. The merit or folly of larger field sizes is unknown.

Continuous course is thought to be better than split-course because it prevents the re-growth of surviving tumor cells between courses. Very few facts bolsters this theory. The altemating strategy requires split-courses. However, effective chemotherapy may prevent or inhibit proliferation of tumor cells between courses.

Dose can be quite difficult to sort out because the biological equivalent dose of different fractionation schemes is poorly expressed in rad, Gray (Gy) or other physical units. The effects 
on acute-effect tissue (tumors, bone marrow, mucosa) may not be paralleled in late-effect time (brain, lung). NSD and $\alpha / \beta$-ratio models need further testing. The influence of time and chemotherapy on these variables may be quite important, but are presently poorly understood. As a general rule, fraction sizes greater than $2 \mathrm{~Gy}$ are more harmful to late-effect tissue, but kill more acute tissue per session. Conversely, fraction size less than 2 Gy respect late tissue, but kill fewer acute effect tissues per session. Simply comparing total doses amongst reported studies can be quite misleading. With this in mind, Table 3 describes the reported failure by total dose.

Table 1

Limited small cell lung cancer local failure

\begin{tabular}{lllll}
\hline Sequence & Group/Inst. & Chemo & CC & C+TRT \\
\hline Mixed & MGH & V & & $32 \%$ \\
Sequential & Memorial & A & & $35 \%$ \\
& Yale & B & & $3.8 \%$ \\
Altemating & IGR & A & 42 & $25 \%$ \\
& SEG & B & 42 & $23 \%$ \\
Concurrent & SEG & B(A) & 40 & $18 \%$ \\
& CALGB & B & 82 & $37-69 \%$ \\
& NCI & B & 67 & $29 \%$ \\
& SWOG & A & & $29 \%$ \\
& Penn & A & & $4 \%$ (pure) \\
& & & & $100 \%$ (var.) \\
\hline
\end{tabular}

V=varied - most cyclophosphamide-based, some used platinum-based; $A=$-platinum-based; $B=c y c l o p h o s p h a m i d e-$ based.

*2nd randomization for responders to: $\pm A$.

Table 2

Influence of volume

\begin{tabular}{lll}
\hline Large volume: & CALGB & $28-42$ \\
& SWOG & 29 \\
& Penn & 16 \\
\hline
\end{tabular}

\section{Highlighted studies}

Choi retrospectively analyzed 207 patients from the Massachusetts General Hospital between 1974 and 1977 [10]. The TRT dose ranged from 30 to $40 \mathrm{~Gy}$ in the early years, and after 1978 the TRT dose increased from 44 to $52 \mathrm{~Gy}$. Doses of 30 and $35 \mathrm{~Gy}$ had a failure of $100 \%$ and $79 \%$, respectively. The TRT doses between 45 and $50 \mathrm{~Gy}$ had local failure of $39 \%$ and $36 \%$, respectively. The dose $40 \mathrm{~Gy}$ has a local failure of $43 \%$. The authors suggested that these data show a 'dose-response' relationship. Details regarding volume, pre- or post-CC, total time of treatment and method of integration of TRT and CC were not discussed. These data were collected over a decade, with changes in staging, $\mathrm{CC}$ and dose, but the univariate analysis evaluated dose only. Local failure does not seem to improve appreciably above $40 \mathrm{~Gy}$. 


\section{Sequential trials}

Yale [11] used cytoxan, etoposide and methotrexate, an infrequently used CC regimen, for 3 cycles followed by split-course TRT ( $60 \mathrm{~Gy} / 6$ weeks/3 Gy/fraction). The local failure was only $3.8 \%$. However, $42 \%$ had less than CR; 2-year survival was $<30 \%$. Duration of follow-up (minimum or median) was not mentioned. Only 7/26 patients remained alive. We know one local failure (? from CR), but status of the other 25 patients remains in doubt, and durability of the local control in the 7 survivors remains in question. TRT volume, pre- or post-chemotherapy, use of spinal cord blocks, and methods of assessment of local control were not discussed. Furthermore, ultimate local control, as opposed to first failure, would be noteworthy.

The Memorial Sloan-Kettering developed the PE regimen, alternated it with CAV, prior to $45 \mathrm{~Gy}$ continuous course, 4 days/week using $2.5 \mathrm{~Gy}$ fractions. The target volumes were determined by CT scan, using multiple-field techniques ( 3 fields, some laterals). Whether volumes were pre- or post-chemotherapy was not reported. Of 34 patients, 12 achieved CR (35\%) and 18 achieved PR (53\%) after CC, and increased to 65\% CR and 26\% PR after TRT. Four failed infield, 3 outside the field and 2 both. Local failure was 29\%, and local control was 65\% (22/34). However, there were 9 local failures, but it is not clear what denominator is appropriate. Since the initial CR rate to chemotherapy was only $35 \%$ and $65 \%$ after TRT, longer follow-up may have yielded more locoregional failures, but that data was not provided.

Table 3

Influence of dose in local failure: selected series

\begin{tabular}{|c|c|c|}
\hline Dose & Institution & $L F(\%)$ \\
\hline$<40$ & MGH & $79-100$ \\
\hline \multirow[t]{3}{*}{40} & MGH & 43 \\
\hline & $\mathrm{NCI}$ & 29 \\
\hline & SEG & 23 \\
\hline \multirow[t]{5}{*}{45} & MGH & 39 \\
\hline & Mennorial & 29 \\
\hline & SWOG & 29 \\
\hline & SEG & 18 \\
\hline & Penn & 16 \\
\hline \multirow[t]{3}{*}{50} & CALGB & $28-42$ \\
\hline & MGH & 36 \\
\hline & IGR & 25 \\
\hline 60 & Yale & $3.8(?)$ \\
\hline
\end{tabular}




\section{Alternating schemes}

The first SEG and the Institute Gustave Roussy (IGR) both used CC on week 1 of cycles followed by large fraction ( $2.5-3.0 \mathrm{~Gy}$ ), split-course TRT to total doses of $40 \mathrm{~Gy}$ (weeks 5,8 and 11) for the SEG trial and 45-55 Gy (weeks 6, 12, 15) for the IGR. The SEG trial was randomized, the CC group failed in $42 \%$, but the TRT group failed in $23 \%$. The IGR reported $12 \%$ failure in the chest alone, but $25 \%$ in chest plus other sites. The technical factors were not discussed by the SEG, but the IGR did boost off cord for $15 \mathrm{~Gy}$ with lateral or angled fields.

\section{Concurrent trials}

The second SEG, Cancer and Leukemia Group B (CALGB), and National Cancer Institute (NCI) all used cyclophosphamide-based CC and concurrent TRT. The NCI and CALGB used continuous courts for 3 and 5 weeks, respectively, but the SEG used split-course with TRT administered in $3 \mathrm{~Gy}$ fractions during weeks 1,2 and 4 . The NCI used $40 \mathrm{~Gy}$ in 3 weeks $(2.66$ Gy/fraction) and wide posterior spinal cord shields. Failure in the chest was recorded at $67 \%$ for the CC group versus $29 \%$ for the TRT group. Variant histology was excluded from the NCI trial. The CALGB used $50 \mathrm{~Gy}$ in 5 weeks $(2.0 \mathrm{~Gy} /$ fraction), using a large volume technique, which included the entire mediastinum plus the supraclavicular fossa. There were 3 arms: (1) immediate TRT; (2) delayed TRT; and (3) not TRT. The chest-only and chest plus distant metastasis (DM) was: (1) $28 \%$ and $37 \%$; (2) $42 \%$ and $69 \%$; and (3) $67 \%$ and $82 \%$. The concurrent SEG trial used 45 Gy during the first two CAV cycles. The addition of TRT did not improve survival. A subsequent randomization to $\mathrm{PE}$ improved survival in those randomized. The local failure of this trial was $40 \%$ in the CC group versus $18 \%$ in arms randomized to TRT. A retrospective comparison between the SEG alternating and concurrent trials showed a local and DM of $22 \%$ versus $35 \%$, respectively, $P=0.01$; however, there was no difference for local only [12];

\section{Concurrent PE and TRT}

The Southwest Oncology Group (SWOG) and University of Pennsylvania (Penn) have both used PE regimens (not identical) and $45 \mathrm{~Gy}$ TRT. The SWOG gave $45 \mathrm{~Gy}$ in 5 weeks. The SWOG trial used generous fields with $2-2.5 \mathrm{~cm}$ margins including the entire mediastinum, contralateral hilum and both supraclavicular fossae. The off-cord technique is not described. Penn gave $45 \mathrm{~Gy}$ in 3 weeks using $1.5 \mathrm{~Gy}$ fractions Bid, CT planning and avoiding direct posteriorspinal cord blocks. Penn's fields were 'tight' with a margin of 1.0-1.5 cm. Average field size was $13.5 \times 16.5$ (range $11-17.5 \mathrm{~cm} \times 12-26.5 \mathrm{~cm}$ ). The contralateral hilum was never treated. Only $45 \%$ had ipsilateral supraclavicular fossa treated. The SWOG will report on 154 patients with $12 \%$ chest or chest and brain failure, but 26 less than PR patients (17\%) are not scored as failures. The Penn trial had only $2 / 31(6 \%)$ with less than CR. With a median follow-up of 42 months, $5 / 31(16 \%)$ have failed locally. All had variant histology (415 presented with variant histology). For pure SCLC, the total failure was $4 \%$ (1/27), and zero for those receiving full dose TRT.

In conclusion, studies vary in how they report local control and failure. Although multiple variables may be important, most authors isolate variables chosen for discussion. No convincing data points to a dose-response relationship above $40 \mathrm{~Gy}$. The issue of sequence and type of CC are linked to this issue; definitively for toxicity, but probably response too. There are no studies addressing pre- versus post-chemotherapy volume or large volume vs small volume. Technique 
of lung and spinal cord protection influence results, but no study specifically addressed this issue. The larger volume, larger dose, larger margin studies do not appear to have better survival or local control than the small study, which intentionally avoids the supraclavicular and contralateral hilar nodes.

These issues would probably not warrant a prospective trial, but carefully designed trials can identify these factors and report their influence.

\section{References}

1 Turrisi, A.T. (1988) The role of radiotherapy for limited small cell lung cancer. Oncology, 2: 19-25.

2 Turrisi, A.T. (1988) Combined-modality treatment of limited small cell lung cancer. Adv. Oncol., 4: 17-25.

3 Turrisi, A.T. (1989) Combined-modality therapy for limited small cell lung cancer: a decade of improvement. In: Small Cell Lung Cancer and Non Small Cell Lung Cancer. (R.J. Gralla and L.H. Einhorn eds.), Royal Society of Medicine Services, pp. 53-61.

4 Perez, C.A., Einhorn, L., Oldham, R.K. et al. (1984) Randomized trail of radiotherapy to the thorax in limited small-cell carcinoma of the lung treated with multiagent chemotherapy and elective brain irradiation: a preliminary report. J. Clin. Oncol., 2: 1200-1208.

5 Bunn, P.A., Jr., Lichter, A.S., Makuch, R.W. et al. (1987) Chemotherapy alone or chemotherapy with chest radiation therapy in limited stage small cell lung cancer. Ann. Intern. Med., 106: 655-662.

6 Perry, M.C., Eaton, W.L and Proper, K.J. (1987) Chemotherapy with or without radiation therapy in limited small-cell carcinoma of the lung. N. Engl. J. Med., 316: 912-918.

7 Souhami, R.L., Geddes, D.M., Spiro, S.G. et al. (1984) Radiotherapy in small cell cancer of the lung treated with combination chemotherapy: a controlled trial. Br. Med. J., 288: 1643-1646.

8 Østerlind, K., Hansen, H.H., Hansen, H.S. et al. (1986) Chemotherapy versus chemotherapy plus irradiation in small cell lung cancer. Results of a controlled trial with five years follow-up. Br. J. Cancer, 54: 7-17.

9 Comis, R.L. (1982) Small cell carcinoma of the lung. Cancer Treat. Rev. 9: 237.

10 Choi, N.C. and Carey, R.C. (1987) Loco-regional failure rate in relation with radiation dose in combined modality approach of multiagent chemotherapy and radiotherapy for limited stage small-cell lung carcinoma. Int. J. Radiat. Oncol. Biol. Phys., 13 (Suppl. 1): 188.

11 Papac, R.J., Son, Y., Bien, R. a al. (1987). Improved local control of thoracic disease in small cell lung cancer with higher dose thoracic irradiation and cyclic chemotherapy. Int. J. Radiat. Oncol. Biol. Phys., 13: 993-998.

12 Birch, R., Omura, G., Greco, F.A. et al. (1988). Pattems of failure in combined chemotherapy and radiotherapy for limited small cell lung cancer: Southeastem Cancer Sudy Group experience. NCI Monogr., 6: 265-270. 